\title{
Reducing the radioactive doses of liquid samples taken from reprocessing plant vessels by volume reduction
}

\author{
G. Janssens-Maenhout ${ }^{\mathrm{a}, *}$, J. Buyst $^{\mathrm{b}}$, P. Peerani ${ }^{\mathrm{a}}$ \\ ${ }^{a}$ Institute for the Protection and the Security of the Citizen, Joint Reserach Centre Ispra, Via E. Fermi, 1, I-21020 ISPRA, Italy \\ ${ }^{\mathrm{b}}$ University of Ghent, Faculty of Engineering, TW08 Technologiepark, B914, B-9052 ZWIJNAERDE, Belgium
}

Received 3 March 2006; accepted 26 September 2006

\begin{abstract}
Size reduction is attractive because it should enable a solution to be analysed by much more direct, and therefore faster and simpler, methods. For instance if the traditional $7 \mathrm{ml}$ vials used in reprocessing plants can be replaced by vessels containing less than $1 \mu l$, it should be possible to analyse the non-diluted solutions in gloveboxes. These vessels would be electro-mechanical, so the term MEMS might be appropriate.

This paper determines a conservative estimate for the dose reduction that would be obtained if microlitre samples were extracted from an input accountancy tank at a reprocessing plant, in which the spent fuel is dissolved in nitric acid. This estimate has to take into account the self-shielding effect, that varies for different low-energy and high-energy gamma-emitting isotopes. The typical composition of the solution from an input accountancy tank in a reprocessing plant is first derived by means of a burn-up code. Eight different spent fuel cases are considered to cover the range of fission products, that can emit low and high energy gamma's. The neutron and gamma fluxes emitted from the classical $7 \mathrm{ml}$ vial and from a vessel with less than a microlitre solution are calculated by means of Monte Carlo simulations. The resulting doses are calculated and compared in average and in distribution for different cases of spent fuel composition. For a volume size reduction of 6300 an averaged conservative dose reduction of 6000 is obtained.
\end{abstract}

(C) 2006 Elsevier B.V. All rights reserved.

PACS: MCNP; SCALE; ORIGEN-S

\section{Introduction}

\subsection{Challenges for the on-site sample characterization}

In the nuclear fuel cycle, conversion, enrichment, fuel fabrication and reprocessing plants are the most vulnerable facilities from a proliferation point of view, because the nuclear material (mainly $\mathrm{U}$ and $\mathrm{Pu}$ ) in these facilities is directly accessible in liquid, gas or powder form, and are therefore easier to divert than in, e.g. a reactor or in spent fuel storage. The provision of safeguards measures have been more elaborate in reprocessing and MOX fuel fabrication plants, because only these facilities deal with $\mathrm{Pu}$ in liquid and powder form.

In reprocessing plants there exist two methods for determining the nuclear material inventory in accountancy tanks: either by a combination of an accurate measurement of the total vol-

\footnotetext{
* Corresponding author. Tel.: +390332 78 5831; fax: +390332789216.

E-mail address: greet.maenhout@jrc.it (G. Janssens-Maenhout).
}

ume at the operating conditions and a precise sample analysis in g/l (e.g. with hybrid K-edge) or by combining the total weight of the tank inventory with an isotope dilution mass spectrometry $(\mathrm{g} / \mathrm{g})$ for sample characterisation. The first method has found more common practice, because it provides a higher throughput of samples in the analytical laboratory. As reported by Daures et al. (2003), on-site analytical laboratories have been developed to provide the fast laboratory responses that are needed for near real-time accountancy.

The sample composition is typically $3-5 \mathrm{M}$ nitric acid in which per litre up to $250 \mathrm{~g}$ spent fuel is dissolved, leading to densities of $1.4 \mathrm{~kg} / \mathrm{l}$. Nowadays a sample of the accountancy tanks' solution is automatically sucked into a vial, that travels through a transfer line directly to the neighbouring analytical laboratory. Even with on-site laboratories, the time to report a sample analysis is affected by extensive sample preparation, for instance a pre-separation or a dilution with a factor 20,000 is needed before the sample can be analysed in a glove box. A considerable simplification in sample preparation will shorten, substantially, the measurement procedure. This simplification can be obtained 
by miniaturisation of the sample, as demonstrated in numerous applications in the pharmaceutical and biochemical industrial research.

\subsection{Micro-vessels instead of milli-vials}

The device envisaged here would lead to reduced radiation, which implies the advantages:

- A direct analysis on the real solution can take place without the need for dilution, in particular direct results allow an interpretation with a smaller uncertainty range.

- The analysis could take place in a glove box, no hot cell is needed and the analytical device would be more transportable because less shielding would be needed.

- The radioactive waste would also be reduced, not only because of the volume of the microchip itself but mainly because of the reduction or even omission of the sample preparation.

\subsection{The basic design}

The sample has to be characterised in concentration and in isotopic composition by photospectrometry. The design must be a compromise between the requirements for manufacturing, those for reproducible spectrophotometric measurements and those for the special treatment of the radioactive solutions. Lascola et al. (2002) have reported on all requirements for reproducible photospectrometry of $\mathrm{U}$ nitric acid solutions. As characterised by Gad-el Hak (1999), the microelectromechanical system (MEMS) consists of a microchip, combining an actuator with a sensor and a microprocessor, that are equipped with a micro power supply input and a communication output. The MEMS envisaged here would be somewhat different.

As shown in Fig. 1 the MEMS base consists of three microchannels, all with $300 \mu \mathrm{m} \times 300 \mu \mathrm{m}$ cross section. The first channel contains the reference solution, the middle one is the optical positioning channel and the third represents the chemical analysis channel, through which the subject radioactive solution is pumped based on the principle of electrophoresis. In a cover of quartz cylindrical holes with a diameter of $1000 \mu \mathrm{m}$ are bored as inlets and outlets. Between the two layers a series of electrodes are inserted to create an electric field along the microchannel length axis for the electrophoretic peristaltic movement of the acid solution into the channel. The complete MEMS is embedded in a positioning frame and kept at constant temperature by means of a cooling Peltier element. As substrate material for the base a high density polyethylene (HDPE) has been selected because this polymer is:

- resistant to acidity and irradiation during 3 months; ${ }^{1}$

\footnotetext{
${ }^{1}$ EURATOM requires the storage during 2 months for eventual re-evaluation measurements in case of differences between inspector's results and operator's declarations.
}

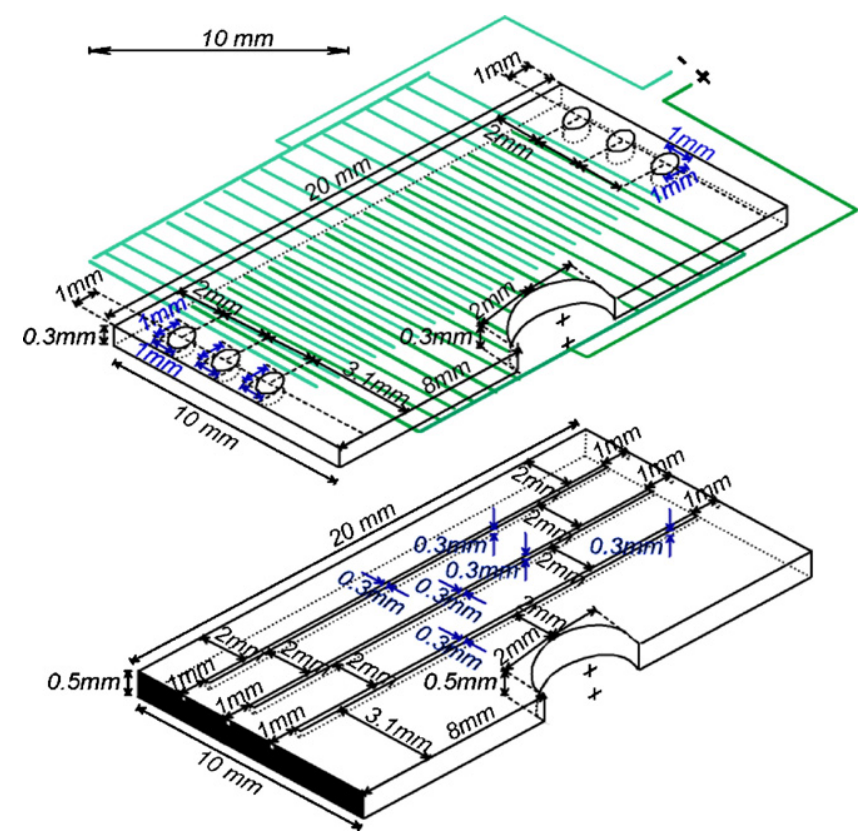

Fig. 1. Design of the microchip for analysing $3 \mathrm{M}$ nitric acid solutions, in which spent fuel is dissolved.

- transparent or translucent for the photospectrometric analysing technique;

- compatible with the material requirements for hot embossing.

The feasibility of the photospectrometry on those microchips has been demonstrated by Nucifora (2004). The fluiddynamic aspects for filling the microchannel with the radioactive acid solution has been discussed by Macerata (2004). The thermal effects of the radioactive hot solution has been analysed by Uyttenhove (2005) and the fluiddynamic aspects of the eletrophoretic flow with proof of homogeneity has been demonstrated by Matthews (2005).

\subsection{Dose calculation for the microchip}

The incentive for scaling down the vial is that it will enable the handling of the filled MEMS without the extra shielding provided by a hot cell. Thus it is important to determine the upper limit of dose to which an operator, handling the microchip in a glove box is exposed. Although an almost proportional scaling down of the dose with sample size is expected, the vanishing self-shielding effect in the microchip leads to a higher dose. This is quantified in this work for different cases of spent fuel by determining, in a conservative way, the ratio of dose reduction versus volume reduction.

First the specific isotopic composition of a solution from the input accountancy tank is modelled with a burn-up code, for different types of spent PWR fuel assemblies, the histories of which are specified in Table 1. This modelled solution then forms the basis for the neutron/photon transport calculations in the Monte Carlo simulations performed for both the vial and MEMS geometries. The radioactive doses for a traditional vial of $7 \mathrm{ml}$ and for a MEMS with only $0.6 \mu l$ at contact, and at a distance 
Table 1

Spent fuel models: seven variants on the reference case

\begin{tabular}{llll}
\hline Case & $\begin{array}{l}\text { Enrichment } \\
(\mathrm{wt} \% \text { U-235) }\end{array}$ & $\begin{array}{l}\text { Burn-up } \\
\text { (MWday/t) }\end{array}$ & $\begin{array}{l}\text { Cooling period } \\
\text { (days) }\end{array}$ \\
\hline Reference & 3.8 & 43,200 & 3650 \\
Low enrichment & 3.2 & 43,200 & 3650 \\
High enrichment & 4.2 & 43,200 & 3650 \\
Low burn-up & 3.8 & 36,000 & 3650 \\
High burn-up & 3.8 & 64,800 & 3650 \\
Short cooling & 3.8 & 43,200 & 1825 \\
Long cooling & 3.8 & 43,200 & 5475 \\
Extreme cooling & 3.8 & 43,200 & 7300 \\
\hline
\end{tabular}

of $1 \mathrm{~m}$ in air, have been compared for the different spent fuel solutions that are specified in Table 1.

\section{Set-up of the radiation source}

\subsection{Isotopic composition of the modelled spent fuel solutions}

A fuel assembly with an enrichment of $3.8 \mathrm{wt} \%$ of U-235 and with an average power of $30 \mathrm{MW}$ during three identical cycles of 480 days, corresponding to an average burn-up of 43,200 MWday/t fresh $\mathrm{UO}_{2}$ at end of life, and then allowed to cool for 10 years was selected as the reference case. Seven different variants were modelled as indicated in Table 1 to investigate the influence of the initial enrichment grade, the average burn-up and the cooling down period. With the burn-up code, SCALE, the complete fuel depletion was analysed with a 1D neutronics model for the PWR fuel, with a normalised active fuel length representing $1 \mathrm{t}$ of fresh fuel ${ }^{2}$, and with decay calculations preformed with the burn-up dependent cross section libraries that were contained in the ORIGEN-S module. The burn-up history was modelled by repeating a sequence of burn-up steps. The resulting isotopic compositions of each of the specified spent fuels are shown in Fig. 2.

The nuclides in the spent fuel assembly that account for a cutoff percentage of $0.05 \%$ are: U-238, contributing for over $90 \%$ to the total mass, U-235, Pu-238, Pu-239, Pu-240, Pu-241, Pu-242. In all eight cases of spent fuel the fission products contribute for almost $5 \%$ of the total mass and the actinides for a maximum $0.7 \%$. A significant impact of increasing burn-up can be noticed by the reduction of the $\mathrm{U}$-isotope and the enlarged production of fission products.

\subsection{Neutron and gamma source for the modelled solutions}

The major contributors to neutron and gamma emission were now focussed on, in order to model an appropriate neutron and gamma source for the spent fuel solution.

For the neutron source $\mathrm{Cm}-244$ was found to contribute over $96 \%$ to the neutron production for all eight cases. The remaining

\footnotetext{
${ }^{2}$ As shown by Buyst (2005) all variants could be modelled with the same active fuel length as the variation was negligible for the different cases.
}

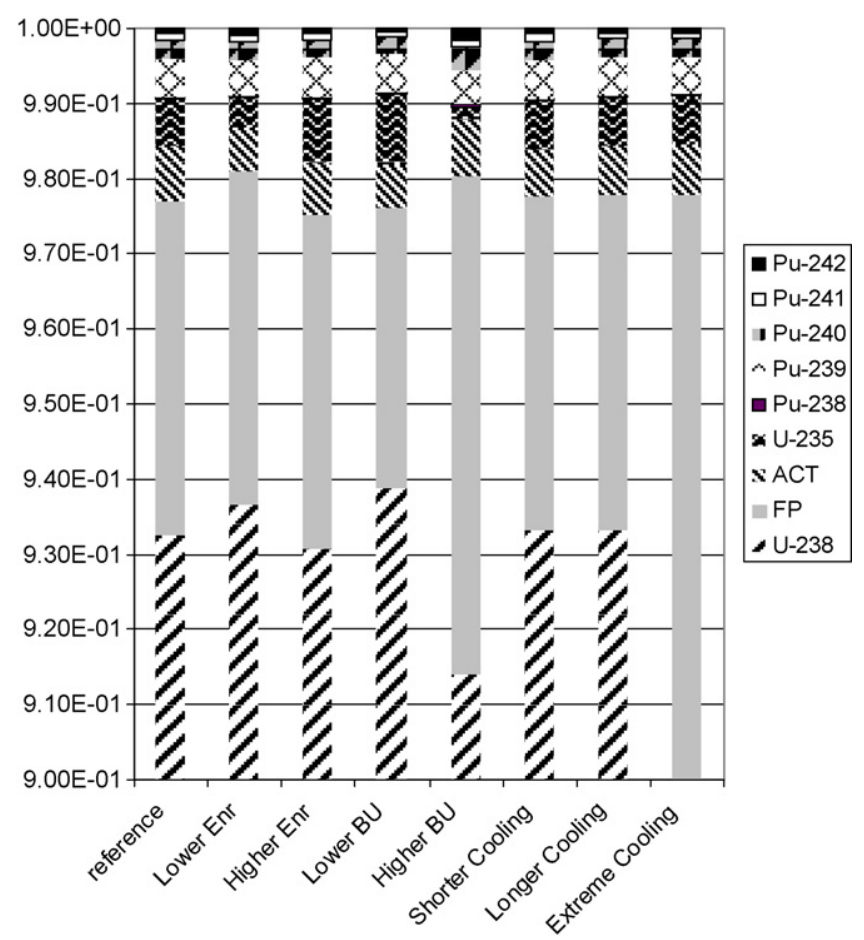

Fig. 2. Calculated isotopic composition for the eight different spent fuel cases of Table 1 .

percentage of neutrons was mainly generated by $(\alpha, n)$ reactions with $\mathrm{Pu}-238, \mathrm{Am}-241$ and $\mathrm{Cm}-244$. The total spectrum of neutrons can be composed by the sum of all spontaneous fission spectra. That has been modelled within an accuracy of $2 \%$ by the spectrum of $\mathrm{Cm}-244$, renormalised with the total neutrons, as shown in Fig. 3. The energy-dependence is described with the Watt-distribution formula for the $\mathrm{Cm}-244$ spectrum. An extensive description is given by Buyst (2005) and full justification of the model can be found in the appendix.

For the gamma source, the fission products represent a hundred times larger source of photons than the actinides, as detailed in Table 2. The Ba- $137 \mathrm{~m}$ appears to contribute $75 \%$ to the gamma spectrum for all cases, except for the case, which had a short cooling period. In the latter case the gamma contribution of $\mathrm{Ba}-$ $137 \mathrm{~m}$ is limited to $50 \%$ due to the fast decay of Cs-134. The following must be summed to explain $99 \%$ of all emitted photons: Ba-137m, Ce-134, Eu-154, Sb-125 and Rh-106, and in the case of long and extreme long cooling also Am. The gamma

Table 2

Total number of particles per second and per $t$ spent fuel as source for MCNP

\begin{tabular}{llllll}
\hline & \multicolumn{2}{l}{ Fission products } & & \multicolumn{2}{l}{ Actinides } \\
\cline { 2 - 3 } \cline { 6 - 6 } & $\gamma(\mathrm{s})$ & $\mathrm{MeV}(\mathrm{s})$ & & $\gamma(\mathrm{s})$ & $\mathrm{MeV}(\mathrm{s})$ \\
\hline Reference & $9.27 \times 10^{15}$ & $3.15 \times 10^{15}$ & & $9.57 \times 10^{13}$ & $2.66 \times 10^{12}$ \\
Low enrichment & $9.10 \times 10^{15}$ & $3.16 \times 10^{15}$ & & $1.03 \times 10^{14}$ & $2.80 \times 10^{12}$ \\
High enrichment & $9.35 \times 10^{15}$ & $3.14 \times 10^{15}$ & & $9.16 \times 10^{13}$ & $2.59 \times 10^{12}$ \\
Low burn-up & $7.76 \times 10^{15}$ & $2.56 \times 10^{15}$ & & $7.18 \times 10^{13}$ & $2.13 \times 10^{12}$ \\
High burn-up & $1.36 \times 10^{16}$ & $4.95 \times 10^{15}$ & & $2.02 \times 10^{14}$ & $4.43 \times 10^{12}$ \\
Short cooling & $1.57 \times 10^{16}$ & $5.67 \times 10^{15}$ & & $8.18 \times 10^{13}$ & $2.00 \times 10^{12}$ \\
Long cooling & $7.61 \times 10^{15}$ & $2.46 \times 10^{15}$ & & $1.06 \times 10^{14}$ & $3.17 \times 10^{12}$ \\
Extreme cooling & $6.61 \times 10^{15}$ & $2.12 \times 10^{15}$ & & $1.14 \times 10^{14}$ & $3.55 \times 10^{12}$ \\
\hline
\end{tabular}




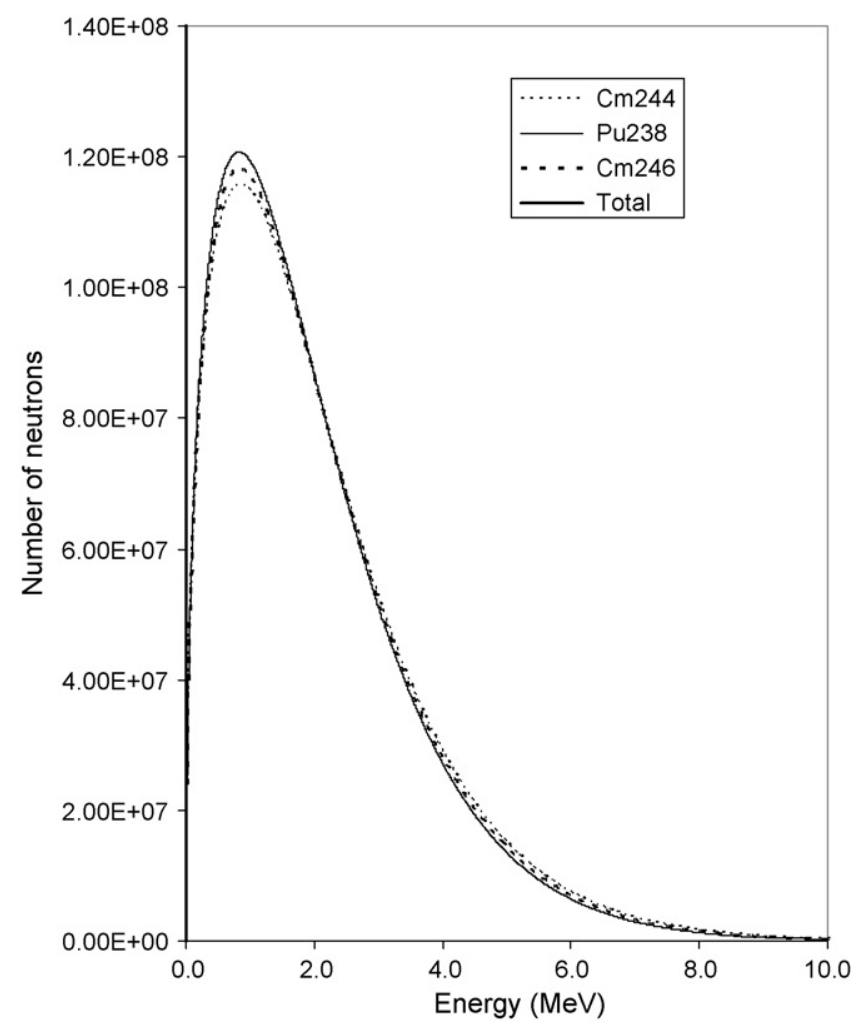

Fig. 3. Cm-244 spectrum vs. the total spectrum of the three most contributing nuclides for the reference case, that is representative for all the modelled cases of spent fuel solutions.

spectrum has been modelled within an accuracy of $0.3 \%$ by the discrete gamma spectrum composed of the spectra of the three most irradiant isotopes, that is renormalised with the total generated photons. More details on the justification of the gamma source model are extracted from Buyst (2005) and are given in the appendix.

\section{Dose calculations}

Given the fact that the sample radioactive solution is enclosed in HDPE material, the dose at contact with the HDPE, and the dose at a distance of $1 \mathrm{~m}$ away from the sample, should only be attributable to the neutron and gamma fluxes. The dose calculation was based on the flux-to-dose factors for neutrons and photons obtained from the 1977 ANSI/ANS and NCRP-38 databases.

The simulations were performed with the geometries as specified in Fig. 4 with materials densities of: $1.1 \mathrm{~g} / \mathrm{cm}^{3}$ for rubber, $0.96 \mathrm{~g} / \mathrm{cm}^{3}$ for HDPE, and $2.65 \mathrm{~g} / \mathrm{cm}^{3}$ for quartz. The millivial contained $7 \mathrm{ml}$ solution and MEMS contained $0.636 \mu \mathrm{l}$ solution in the analysis channel and $0.236 \mu l$ in the inlet and in the outlet. The solution concentration was stepped from 2-25 to $100-250 \mathrm{~g} / \mathrm{l}$. The neutron and photon sources were modelled for each of the eight cases with mean values as specified in Table 3.

\subsection{MEMS results}

The contact dose for the most conservative case, filled with $2 \mathrm{~g} / \mathrm{l}$ nitric acid solution with spent fuel reaches $7.93 \mu \mathrm{Sv} / \mathrm{h}$. This
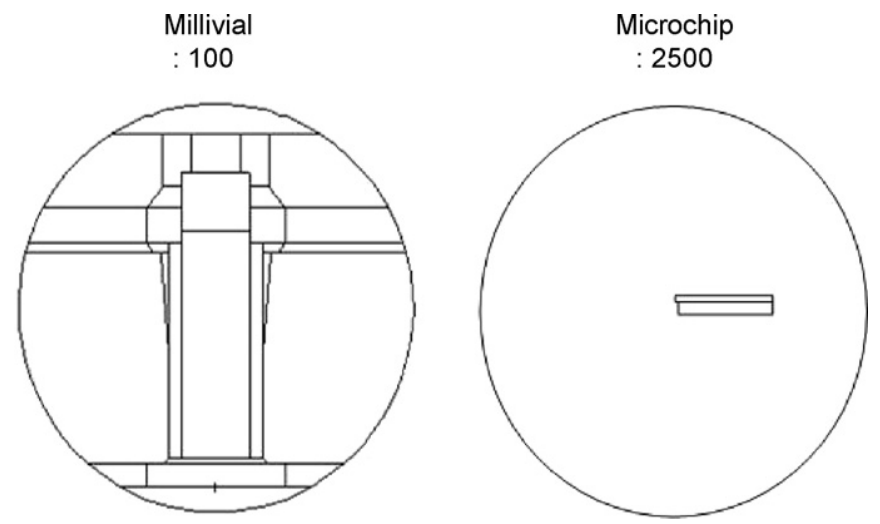

Fig. 4. Geometry applied in the MCNP calculations for the millivial (scale 1 over 1/100) and for the microchip (scale 1 over 1/25,000).

Table 3

Total number of particles per second and per $t$ spent fuel as source for MCNP

\begin{tabular}{llc}
\hline Case & Photons $(\gamma / \mathrm{s} / \mathrm{t})$ & Neutrons $(\mathrm{n} / \mathrm{s} / \mathrm{t})$ \\
\hline Reference & $4.00 \times 10^{8}$ & $7.24 \times 10^{15}$ \\
Low enrichment & $5.70 \times 10^{8}$ & $7.16 \times 10^{15}$ \\
High enrichment & $3.21 \times 10^{8}$ & $7.28 \times 10^{15}$ \\
Low burn-up & $1.74 \times 10^{8}$ & $6.01 \times 10^{15}$ \\
High burn-up & $2.27 \times 10^{8}$ & $10.9 \times 10^{15}$ \\
Short cooling & $4.82 \times 10^{8}$ & $12.6 \times 10^{15}$ \\
Long cooling & $3.33 \times 10^{8}$ & $5.87 \times 10^{15}$ \\
Extreme cooling & $2.78 \times 10^{8}$ & $5.09 \times 10^{15}$ \\
\hline
\end{tabular}

means that a normal operator in a radiochemical facility could handle the component with a pair of gloves during the complete year, without any fear of reaching the dose limitation for hands. The very small dose at $1 \mathrm{~m}$ also confirms that there is no possibility of reaching the maximum effective dose limitation, for the total body, for a single year $(20 \mathrm{mSv} / \mathrm{year}$ and in the near future $12 \mathrm{mSv} /$ year), and for five consecutive years $(100 \mathrm{mSv} /$ 5 year).

The gamma part represents the major contribution to the dose, because the neutron dose is a factor $10^{-5}$ lower than the gamma dose, leading to maximum $1.332 \mu \mathrm{Sv} /$ year. Although the maximum dose is obtained in the case of spent fuel with short cooling, the spent fuel with high burn-up also leads to a relatively high dose. The enrichment grade characteristic shows a minor influence on the results, as shown in Table 4.

Table 4

Results of the dose caused by the gamma flux at contact and at $1 \mathrm{~m}$ distance for a solution of $2 \mathrm{~g} / \mathrm{l}$ nitric acid with dissolved spent fuel

\begin{tabular}{lll}
\hline Case & Dose at contact $(\mu \mathrm{Sv} / \mathrm{h})$ & Dose at $1 \mathrm{~m}(\mu \mathrm{Sv} / \mathrm{h})$ \\
\hline Reference & 4.57 & $1.73 \times 10^{-3}$ \\
Low enrichment & 4.52 & $1.71 \times 10^{-3}$ \\
High enrichment & 4.60 & $1.74 \times 10^{-3}$ \\
Low burn-up & 3.80 & $1.44 \times 10^{-3}$ \\
High burn-up & 6.87 & $2.60 \times 10^{-3}$ \\
Short cooling & 7.93 & $3.01 \times 10^{-3}$ \\
Long cooling & 3.71 & $1.41 \times 10^{-3}$ \\
Extreme cooling & 3.22 & $1.22 \times 10^{-3}$
\end{tabular}

For each of the eight cases of spent fuel, MCNP calculations have been performed twice, with the neutron group spectrum and with a continuous spectrum. 


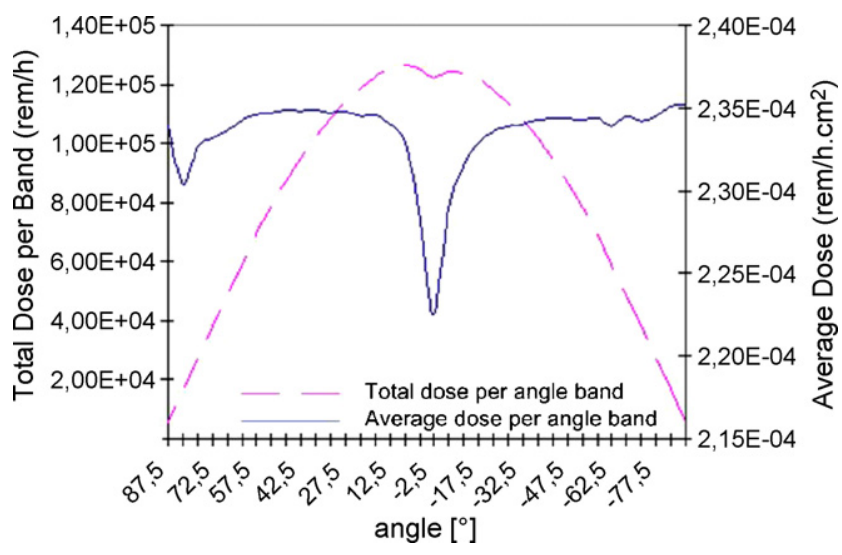

Fig. 5. Distribution of the dose from $+90^{\circ}$ above the mid plane to $-90^{\circ}$ below the mid plane of the microchip (reference case with $2 \mathrm{~g} / \mathrm{l}$ solution).

The dose calculations have been performed with a six-group spectrum for the neutron part, and a discrete spectrum for the gamma part. It was observed that the neutron dose calculations with the continuous spectrum yields an up to $20 \%$ lower dose than those with the group spectrum, which justifies the use of the group spectrum. In contrast, the calculations with the group spectrum and with the discrete spectrum for the gamma dose have shown a difference of less than $1 \%$. This justifies the use of a renormalised $\mathrm{Ba}-137 \mathrm{~m}$ discrete spectrum. More details are given in Tables A1 and A2 of the appendix.

Given the flat geometry of the MEMS, a non-uniform distribution of the dose is expected, which is governed by the photon dose. Fig. 5 shows the angular dependency of the dose, which clearly shows that the particles emitted in the plane of the microchip (at the angle around $0^{\circ}$ ) lead to the lowest dose contribution, because the particles have to penetrate at least $1 \mathrm{~mm}$ of thermalising material. Under an angle substantially different from $0^{\circ}$, the particles can profit from a faster exit out of the thermalising material, up to $0.3 \mathrm{~mm}$ for the upwards directed onces and up to $0.5 \mathrm{~mm}$ for the downwards directed onces.

Two simplified models, that could be easily used in optimisation studies, were evaluated: a point source and a line source with the same intensity. For the neutron dose the simplified line source gives a very small overestimation of only $0.69 \%$ and the point source overestimates by only $0.65 \%$. For the gamma dose the simplification in geometry has a larger impact, the simplified line source gives an overestimation of $2.73 \%$ and the point source overestimates by $2.90 \%$.

\subsection{Dose reduction versus volume reduction}

It has been shown above that the absolute dose at contact should be well below all official dose limitations. Here the minimum percentage of dose reduction obtained by reducing volume size is discussed. To derive the relative dose reduction obtained by volume reduction, the doses emitted at a distance of $1 \mathrm{~m}$ are compared for both the millivial and the proposed device.
Table 5

The reduction of the dose at $1 \mathrm{~m}$ distance for the microchip relative to the millivial has been calculated in function of different common solution concentrations

\begin{tabular}{ccll}
\hline Concentration U-Pu $(\mathrm{g} / \mathrm{l})$ & U-Pu mass $(\mu \mathrm{g})$ & Mass reduction & $R_{\mathrm{D}}$ at $1 \mathrm{~m}$ \\
\hline 2 & 2 & 7000 & 6092 \\
25 & 28 & 6250 & 6046 \\
100 & 111 & 6306 & 5958 \\
250 & 221 & 6335 & 5896
\end{tabular}

The dose reduction factor has been averaged over all eight spent fuel cases.

The volumetric reductions can be accurately calculated by taking into account the actual geometries of both components, assuming that the millivial is systematically filled with $7 \mathrm{ml}$ solution and that there are no gas bubbles when the MEMS is completely filled. The volume of the proposed device is 6321.07 times smaller than the millivial. Given the fact that nowadays, with high burn-up fuel, either a pre-separation or a dilution factor of 20,000 is needed before the sample solution can be analysed, it is obvious that future geometries will be downscaled by this order of magnitude. A further factor of three is feasible with regard to manufacturing, but would require higher electrical forces to fill the micro channel.

To determine the gain in dose reduction for the most conservative case, a dose reduction factor $R_{\mathrm{D}}$ has been defined as the ratio of the lowest dose for the millivial $D_{\mathrm{m}}$ to the highest dose of the MEMS $D_{\mu}$ caused by neutrons $n$ and photons $\gamma$ calculated either with the group spectrum (index $g$ ), or with the renormalised continuous Watt spectrum of $\mathrm{Cm}-244$ neutrons and the renormalised discrete photon-spectrum of Ba-137m (index c), more specifically by

$R_{\mathrm{D}}=\frac{\min \left(D_{\mathrm{m}}^{\mathrm{n}, \mathrm{g}}, D_{\mathrm{m}}^{\mathrm{n}, \mathrm{g}}\right)+\min \left(D_{\mathrm{m}}^{\gamma, \mathrm{g}}, D_{\mathrm{m}}^{\gamma, \mathrm{c}}\right)}{\max \left(D_{\mu}^{\mathrm{n}, \mathrm{g}}, D_{\mu}^{\mathrm{n}, \mathrm{c}}\right)+\max \left(D_{\mu}^{\gamma, \mathrm{g}}, D_{\mu}^{\gamma, \mathrm{c}}\right)}$

The results are for a solution of $2 \mathrm{~g} / \mathrm{l}$ dissolved spent fuel 6092, meaning that $96.4 \%$ of proportional dose reduction with volume size reduction is achieved. No differences larger than $0.1 \%$ could be observed in the results for the eight cases of specified spent fuel. Similar calculations of dose reduction have been carried out for other concentrations and have to be compared with the volume reduction of 6321 . The resulting characteristics (in mass content) with dose reduction and mass reduction for the different types of solutions are given in Table 5. Even at highly concentrated solutions a dose reduction of 5896 for the given volume size reduction is obtained. It can be concluded that for solutions with up to $250 \mathrm{~g} / \mathrm{l}$ dissolved spent fuel, even of high burn-up and short cooling time a minimum percentage of 93.27 of dose reduction with the volume size reduction is guaranteed.

\section{Conclusion}

A specific MEMS for analyzing radioactive solutions of an input solution (with concentrations varying from 2 till $250 \mathrm{~g} / \mathrm{l})$ has been designed with three microchannels of $300 \mu \mathrm{m} \times 300 \mu \mathrm{m}$ cross section and $18 \mathrm{~mm}$ length. One channel contains the reference solution with neodymium, the channel in the centre is the blank and the third channel contains the 
sample. The concentration of the solution is determined by the photospectra of the light transmitted along the channel axis and absorbed at nuclide-specific wavelengths.

The dose reduction by volume size reduction has been determined by Monte Carlo simulations. If the volume of a typical input solution sample is reduced with a factor 6321 (for the proposed MEMS design) a dose-reduction of 5896 is achieved for the highly concentrated solution of $250 \mathrm{~g} / \mathrm{l}$. The dose reduction is almost proportional with the size reduction for the different types of fuel (UO2 and MOX) and for a burn-up varying from 36,000 to $64,800 \mathrm{MWday} / \mathrm{t}$, which confirms that the change in self-shielding for the low energy $\gamma$-rays is very small.

\section{Appendix A. The microchip dose calculations with MCNP}

\section{A.1. Model of neutron and gamma source}

In order to create an efficient time-dependent neutron source model for the Monte Carlo simulations, it was investigated if the sum of all spontaneous fission spectra cannot be replaced by the spectrum of the most contributing nuclide $\mathrm{Cm}-244$. As shown in Fig. 3 the $\mathrm{Cm}-244$ spectrum did not differ more than $2 \%$ from the cumulative spectrum of the three most contributing nuclides (Cm-244, Pu-238 and Cm-246). This justified to take the Cm244 spectrum, renormalised with the total neutrons (generated by spontaneous fission and by $(\alpha, n)$ reactions) as input to model the neutron source for the input accountancy solution. In addition Fig. A1 shows that the theoretical decay of $\mathrm{Cm}-244$ in a cooling period of minimum 900 days corresponds to the decrease in neutron generation that was calculated with SCALE.

Concerning the energy-dependence of the neutron source model, the application of the continuous Watt-distribution spectrum of $\mathrm{Cm}-244$ was evaluated. In order to guarantee that the continuous Cm-244 spectrum gives a conservative estimation of the neutron source, it is needed to compare the calculated group spectrum with the theoretical group spectrum, derived by integrating the continuous spectrum over the energy intervals as defined for the SCALE simulations. Fig. A2 shows that the sec-

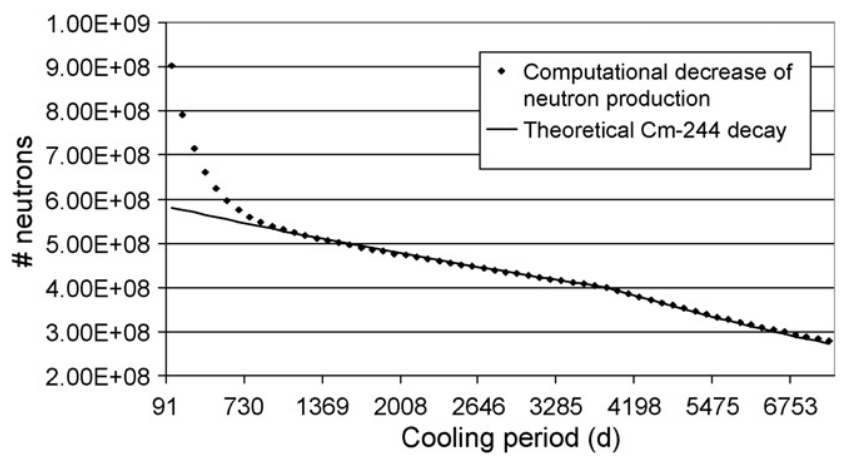

Fig. A1. The simulated neutron generation decreases with time compared to the theoretical decay of $\mathrm{Cm}-244$.

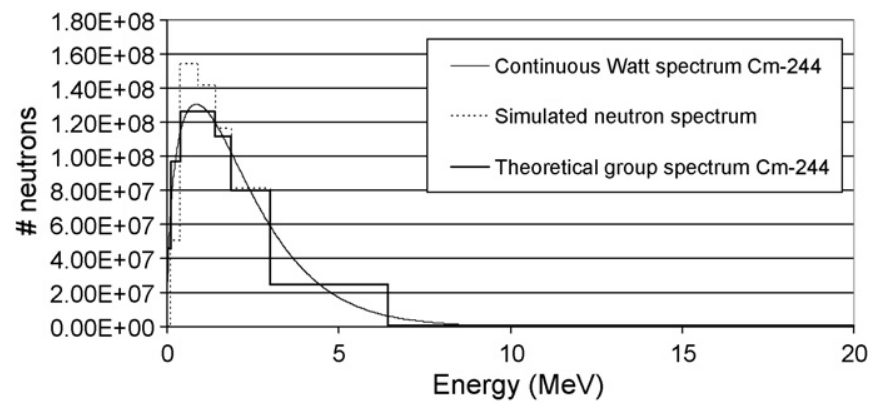

Fig. A2. Neutron spectrum as input: continuous Watt spectrum and simulated group spectrum.

ond and third energy group are not modelled in a conservative way by the theoretical spectrum. Neutrons have been neglected in the lower energy range and substituted by neutrons in the higher energy range in the SCALE calculations. Therefore both cases, the simulated group spectrum and the continuous spectrum have been taken into account for the MCNP calculations.

The gamma group spectrum resulting from the SCALE simulations has been compared to a discrete gamma spectrum composed of the spectra of Ba-137m, Cs-137 and Eu-154, that is renormalised with the total generated photons. The comparison in Fig. A3 allows to create an efficient gamma source model, by replacing the gamma group spectrum with the composed discrete spectrum.

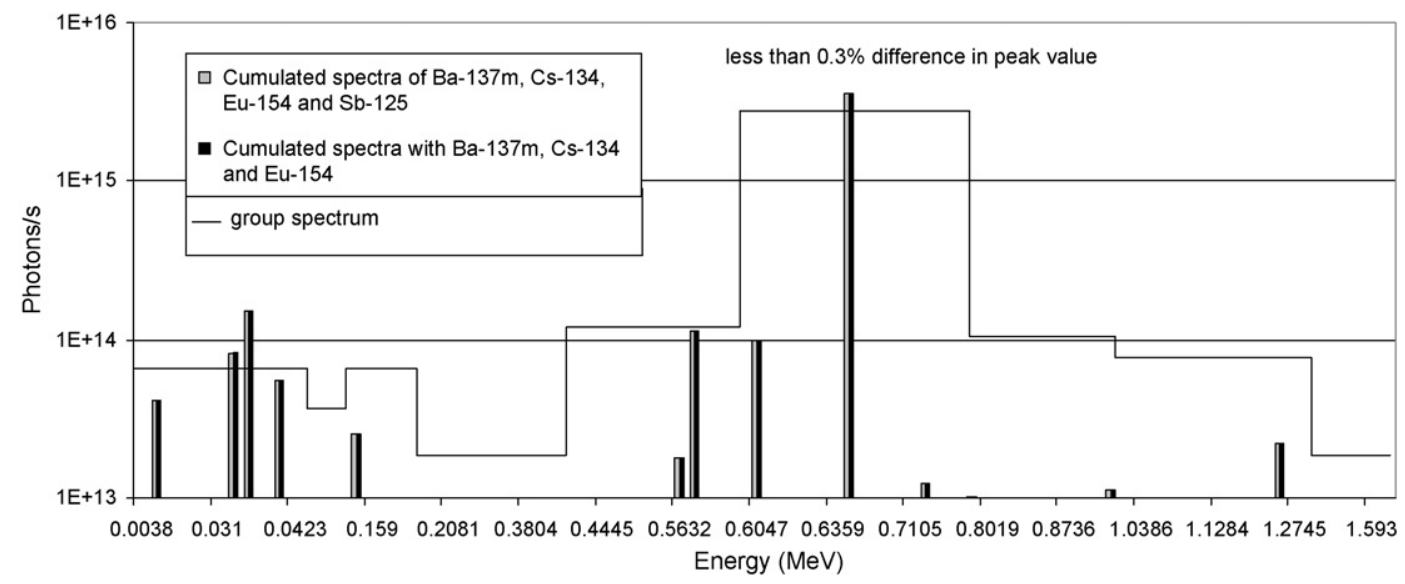

Fig. A3. Comparison of discrete gamma spectra with the three major contributing isotopes and with the four major contributing isotopes, as well as the gamma group spectrum, for the representative reference case. 
Table A1

Results of the dose caused by the neutron flux at contact and at $1 \mathrm{~m}$ distance for a solution of $2 \mathrm{~g} / \mathrm{l}$ nitric acid with dissolved spent fuel

\begin{tabular}{|c|c|c|c|c|}
\hline \multirow[t]{2}{*}{ Case } & \multicolumn{2}{|c|}{ Dose at contact $(\mu \mathrm{Sv} / \mathrm{h})$} & \multicolumn{2}{|l|}{ Dose at $1 \mathrm{~m}(\mu \mathrm{Sv} / \mathrm{h})$} \\
\hline & By group spectrum & By continuous spectrum & By group spectrum & By continuous spectrum \\
\hline Reference & $2.68 \times 10^{-5}$ & $2.23 \times 10^{-5}$ & $1.02 \times 10^{-8}$ & $8.52 \times 10^{-9}$ \\
\hline High enrichment & $2.15 \times 10^{-5}$ & $1.79 \times 10^{-5}$ & $8.20 \times 10^{-9}$ & $6.83 \times 10^{-9}$ \\
\hline Low burn-up & $1.16 \times 10^{-5}$ & $9.69 \times 10^{-6}$ & $4.44 \times 10^{-9}$ & $3.70 \times 10^{-9}$ \\
\hline High burn-up & $1.52 \times 10^{-4}$ & $1.26 \times 10^{-4}$ & $5.79 \times 10^{-8}$ & $4.82 \times 10^{-8}$ \\
\hline Extreme cooling & $1.86 \times 10^{-5}$ & $1.55 \times 10^{-5}$ & $7.10 \times 10^{-9}$ & $5.91 \times 10^{-9}$ \\
\hline
\end{tabular}

For each of the eight cases of spent fuel, MCNP calculations have been performed twice, with the neutron group spectrum and with a continuous spectrum.

Table A2

Results of the dose caused by the gamma flux at contact and at $1 \mathrm{~m}$ distance for a solution of $2 \mathrm{~g} / \mathrm{l}$ nitric acid with dissolved spent fuel

\begin{tabular}{lllll}
\hline Case & \multicolumn{2}{l}{ Dose at contact $(\mu \mathrm{Sv} / \mathrm{h})$} & & \multicolumn{2}{l}{ Dose at $1 \mathrm{~m}(\mu \mathrm{Sv} / \mathrm{h})$} \\
\cline { 2 - 3 } & By group spectrum & By discrete spectrum & & By group spectrum \\
\hline Reference & 4.59 & 4.57 & $1.74 \times 10^{-3}$ & $1.73 \times 10^{-3}$ \\
Low enrichment & 4.56 & 4.52 & $1.74 \times 10^{-3}$ & $1.73 \times 10^{-3}$ \\
High enrichment & 4.60 & 4.60 & $1.43 \times 10^{-3}$ & $1.71 \times 10^{-3}$ \\
Low burn-up & 3.79 & 3.80 & $2.61 \times 10^{-3}$ & $1.74 \times 10^{-3}$ \\
High burn-up & 6.90 & 6.87 & $3.02 \times 10^{-3}$ & $2.60 \times 10^{-3}$ \\
Short cooling & 7.96 & 3.93 & $1.40 \times 10^{-3}$ & $3.01 \times 10^{-3}$ \\
Long cooling & 3.69 & 3.22 & $1.20 \times 10^{-3}$ & $1.41 \times 10^{-3}$ \\
Extreme cooling & 3.19 & &
\end{tabular}

For each of the eight cases of spent fuel, MCNP calculations have been performed twice, with the neutron group spectrum and with a continuous spectrum.

\section{A.2. Results for neutron and gamma source separately}

The dose for the microchip, filled with a solution of $2 \mathrm{~g} / \mathrm{l}$ dissolved spent fuel, that is caused by the neutrons and by the gamma's has been calculated separately for the neutrons and the gamma's and in two different ways, by applying a group spectrum or a continuous spectrum for the neutrons and by a group spectrum or a discrete spectrum for the gamma's. The results are shown in the Tables A1 and A2 below.

\section{References}

Buyst, J. 2005. Nuclear safeguards by lab on the microchip: deterministic calculation of radioactive dose reduction by volume size reduction, Technical Report master thesis for the degree of engineering in physics, University Ghent, Fac. of engineering.

Daures, P., Richir, P., Cremer, B., Ottmar, H., Mayer, K., Blohm-Hieber, U., Decobert, G., Rincel, X., 2003. Commissioning and routine operation of the "laboratoire sur site". In: Proceedings of ESARDA Symposium (Brugge).
Gad-el Hak, M., 1999. The fluid mechanics of microdevices-the freeman scholar lecture. ASME J. Fluids Eng. 121, 5.

Lascola, R.J., Livingston, R.R., Sanders, M.A., McCarty, J.E., Cooper, G.A., 2002. On Line Spectrophotometric Measurement of Uranium and Nitrate in h Canyon, Technical Report WSRC-TR-2002-00334 (rev. 0). West-inghouse Savannah River Company.

Macerata, E. 2004. Micro-fluiddynamic constraints for the lab on the microchip to analyse radioactive solutions, Technical Report master thesis for the degree of nuclear engineering, Polytechnic of Milan, Nucl. Eng. Dep.

Matthews, R. M. 2005. Thermo-fluid-dynamic evaluation of a microelectromechanical device for the analysis of radioactive solutions, Technical Report thesis for the degree of engineering, University Glasgow, Mech. Eng. Dep.

Nucifora, S. 2004. Nuclear safeguards by lab on the microchip: spectrophotometric tests of radioactive solution on microsample, Technical Report master thesis for the degree of nuclear engineering, Polytechnic of Milan, Nucl. Eng. Dep.

Uyttenhove, W. 2005. Thermodynamic evaluation of a microchip to analyse radioactive solutions, Technical Report master thesis for the degree of nuclear engineering, University Ghent, BNEN specialisation. 